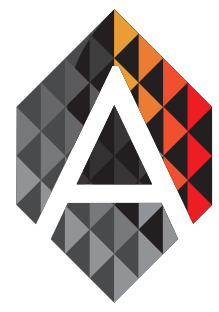

ADCAIJ: Advances in Distributed Computing and Artificial Intelligence Journal Regular Issue, Vol. 7 N. 1 (2018), 67-76

eISSN: $2255-2863$

DOI: http://dx.doi.org/10.14201/ADCAIJ2018716776

\title{
An Agent-based Environment for Dynamic Positioning of the Fogg Behavior Model Threshold Line
}

\author{
Marcus Guimaraes, Diana Adamatti, \\ Leonardo Emmendorfer \\ Federal University of Rio Grande (Brazil) \\ guimaraesmvf@gmail.com, dianaada@gmail.com, leonardoemmendorfer@gmail.com
}

\begin{tabular}{|c|c|}
\hline KEYWORD & ABSTRACT \\
\hline $\begin{array}{l}\text { Persuasive } \\
\text { Technology; } \\
\text { Dynamic } \\
\text { Threshold Line; } \\
\text { Trigger }\end{array}$ & $\begin{array}{l}\text { In this paper, it is presented a mathematical modeling for the action line, or threshold } \\
\text { line, of the Fogg Behavior Model (FBM) as well as an analysis of its positioning in } \\
\text { relation to the dataset. According to the mathematical modeling formation process for } \\
\text { both Motivation and Ability axes, the action line evaluation was performed by simu- } \\
\text { lations via agents. This behavioral model is mainly used as an empirical evaluation } \\
\text { method applied to processes based on persuasive technologies. The results showed } \\
\text { that the threshold line should not be fixed, as originally proposed in the model, but dy- } \\
\text { namically allocated based on the Kolmogorov mean. This dynamic allocation ensures } \\
\text { its use as a visual feature towards greater efficiency in triggers implementations. This } \\
\text { work aims to contribute with an approach that transits between theoretical and prac- } \\
\text { tical when related to applications that requires the FBM, thus allowing the use of this } \\
\text { behavioral model with higher degree of certainty and thus maximizing efficiency in the } \\
\text { evaluation and implementation processes based on persuasive technologies. }\end{array}$ \\
\hline
\end{tabular}

\section{Introduction}

Technology and information can be now considered fundamental on our society (Rahman, 2016). The technological development makes data to be produced almost instantly by a variety of sources. Hence, to transform all that data into knowledge that can be further used by the society is crucial. In this scenario, a mathematical model can be applied in order to describe and predict the individual behavior of a given proportion of society. Consequently, this process is slowed down and becomes inviable to implement large studies based on human interaction. However, with the correct data modeling process, robust algorithms are capable to simulate a social interaction environment (Shannon, 1975) and reduce the high costs of implementation in real life data acquisition processes (Wessels, 2010).

Computational Modeling is an important ally on simulations and social researches. Its characterized as an interdisciplinary area of study where the goal is to promote mathematical and statistical approaches for the development of models and tools capable of representation a relevant phenomena. A direct example of such applicability is the research area known as persuasive technology (Fogg, 2003) which can be described as

Marcus Guimaraes, Diana Adamatti and Leonardo Emmendorfer

An Agent-based Environment for Dynamic Positioning of the Fogg Behavior Model Threshold Line
ADCAIJ: Advances in Distributed Computing and Artifical Intelligence Journal Regular Issue, Vol. 7 N. 1 (2018), 67-76 elSSN: 2255-2863 - http://adcaij.usal.es Ediciones Universidad de Salamanca - CC BY NC DC 
computational entities acting as agents to promote the behavior change and persuasion. In this sense, a mathematical model capable to evaluate different behavior traits in persuasive based process is the Fogg Behavior Model (Fogg, 2009).

A mathematical model is an abstract representation of an object, system or idea (Shannon, 1975). The use of behavior model characterizes an approach based upon improvement of a specific feature by monitoring a dataset. According to the available data, it is possible to take different actions, given the research interest, such as weight control and food intake (Azar et al., 2013), driving speed reduction with young drivers (Bergmans and Shahid, 2013), evaluate and predict natural disasters (Tsai et al., 2015) or improve the trustworthiness of interpersonal relationship (Hongqi et al., 2013). The applicability is uncountable, and this reinforces the importance of adopting a correct mathematical model for evaluate persuasive implementations.

Analysis of behavioral data became a viable option given the ease of data acquisition and applicability. Computer simulations contributions for the development of scientific knowledge is unquestionable. Without these resources, the analysis and interpretation time would increase significantly.

In this paper, we present a topic not properly documented on the literature. The formation process and the threshold line behavior of the FBM given a mathematical modeling of both Motivation an Ability axis. The dynamic threshold line positioning was evaluated with agent-based simulation and it is extremely related to the dataset central tendency properties.

A multi-agent system consists in multiples entities called agents interacting on a shared environment willing to satisfy a common goal. On the other hand, simulation consists on the evaluation of a operational model through time (Uhrmacher and Weyns, 2009). The use of agent-based simulation aims to evaluate the behavior of a set of operations through time characterized by a dynamic application domain.

The modeling process via agents was used in this paper to emulate a social interaction environment capable of satisfying the FBM interactive conditions (Mangina and Carbo, 2010). Hence, it was used the free tool Netlogo ${ }^{1}$.

Section Theoretical Background informs the reader about persuasive technology principles and presents the Fogg Behavior Model. The section Methods describes the mathematical process for both axis formation, considering all FBM variables as well the proposed dynamic threshold line evaluated via agent-based simulation. Finally, results are presented and discussed.

\section{Theoretical Background}

\subsection{Persuasive Technologies}

Thoughts and actions can be influenced by others. The procedure of inducing a set of values and beliefs into another person is called persuasion (Hogan, 2004). Technology has an intrinsic persuasive power, that sometimes can be unnoticed. However, such trait is frequently explored in several areas of our modern society (Larson, 2014). Marketing for instance, applied persuasive strategies willing to improve sales.

Hence, no matter how the information is delivered, internet, television or radio, publicity uses these principles in different intensities (Hogan, 2004).

Persuasive technology can be defined as any interactive computer system designed to change its user behavior. In this context the term Captology or Computer as Persuasive Technologies emerges (Fogg, 2003). Furthermore, any computer device can be used as a persuasive tool. However, in Captology only attitude and intentional behavior changes are considered. These changes must be designed and not a side effect in order to classify a device as persuasive. It is worth mentioning that the persuasive power itself it is a side effect of technology, since computers were not designed with this purpose, but only to manipulate, process and store data (Hillard, 2010). However, persuasive techniques tends to be more effective when applied in an interactive context. This allows the persuasive agent to adjust its tactics throughout the study. Machines do not get tired or frustrated

1. https://ccl.northwestern.edu

Marcus Guimaraes, Diana Adamatti and Leonardo Emmendorfer

An Agent-based Environment for Dynamic Positioning of the Fogg Behavior Model Threshold Line
ADCAIJ: Advances in Distributed Computing and Artifical Intelligence Journal Regular Issue, Vol. 7 N. 1 (2018), 67-76 eISSN: 2255-2863 - http://adcaij.usal.es Ediciones Universidad de Salamanca - CC BY NC DC 
when exposed to negative reactions. Besides, in some situations it is easier to acquire information anonymously trough a technological device rather than using a procedure that involves human interaction (Fogg, 2003).

\subsection{Fogg Behavior Model}

The FBM is mainly used given its applicability on persuasive technology process-based exploration. Essentially consists in two dimension of data corresponding to the level of Motivation and Ability, and a threshold line acting as visual guide for behavior change based on different user stimuli called triggers. We believe that the threshold line or action line presents an intrinsic relation with the type of triggers that can be used in order to promote behavioral change.

Figure 1.a shows the FBM. However, it is not mentioned in the literature a proper placement for the threshold line. It is known that for motivation and ability values above this action line indicates individuals highly receptive for a stimulus called signal trigger. Hence, it can be shown in Fig.1.b that the threshold line placement is intrinsically related with the region corresponding to each type of trigger (Fogg, 2009).

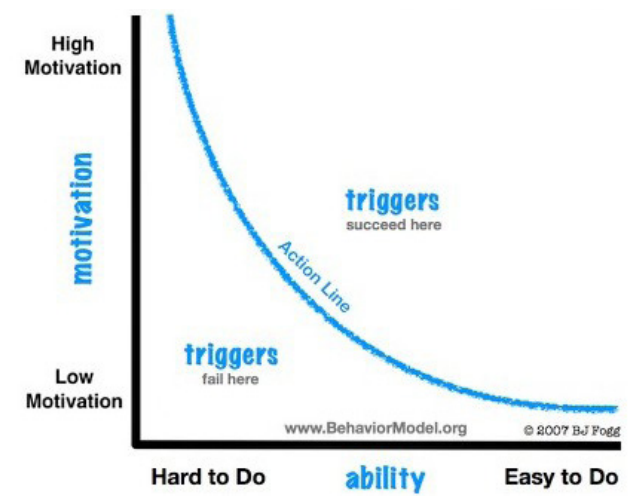

(a)

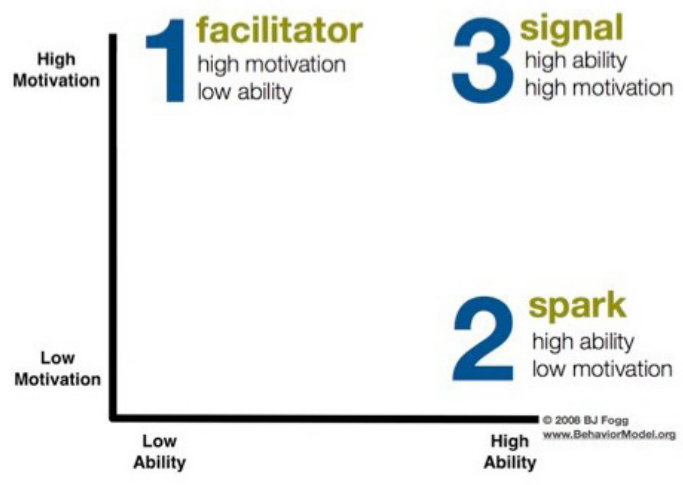

(b)

Figure 1: FBM and the instrinsic relation between the threshold line and triggers (Fogg, 2009).

Hence, as higher Motivation and Ability goes, easier it is to send a stimuli (trigger) to persuade the user. Each axis is formed by six variables. Motivation presents Pleasure, Pain, Hope, Fear, Acceptance and Rejection. On the other hand, Ability presents Time, Money, Physical Effort, Mental Effort, Social Deviance and Routine (Fogg, 2009).

Fogg describes a general and relational equation for the model given by:

$$
B=M A T
$$

Equation (1) can be considered more descriptive then algebraic and it informs that in order to Behavior (B) occur, a interaction between three factors must be in place: Motivation (M), Ability (A) and a stimulus called Trigger (T). Note that a null value for $M A T$ implies $B=0$, which means in the FBM context, no behavior.

\section{Methods}

\subsection{Axis formation process}

The Motivation axis conceptually tries to move the analyzed user up, in order to make it more motivated (Fogg, 2009). As mentioned, all six variables contributes on its formation improving or decreasing its value. Table 1 shows the contribution of each variable on the axis acquisition process:

Marcus Guimaraes, Diana Adamatti and Leonardo Emmendorfer

An Agent-based Environment for Dynamic Positioning of the Fogg Behavior Model Threshold Line
ADCAIJ: Advances in Distributed Computing and Artifical Intelligence Journal Regular Issue, Vol. 7 N. 1 (2018), 67-76 elSSN: 2255-2863 - http://adcaij.usal.es Ediciones Universidad de Salamanca - CC BY NC DC 
Table 1: Variable contribution.

\begin{tabular}{c|c|c|c|c|c}
\hline Pleasure & Pain & Hope & Fear & Acceptance & Rejection \\
\hline+ & - & + & - & + & - \\
\hline
\end{tabular}

Note that three out of six variables contributes increasing the motivation. The other three acts decreasing the axis value. In this paper will be used the following notation:

$m_{1}$ : Pleasure, $m_{2}$ : Hope, $m_{3}$ : Acceptance, $m_{4}$ : Pain, $m_{5}$ : Fear, $m_{6}$ : Rejection.

Hence, two distinct groups can be create in order to categorize all axis variables. one ground containing only variables which contributes with the increase of Motivation, and another group with negative variables.

The Ability (A) axis contains six variables. Its behavior is analogous to the Motivation axis. Table 2 shows each variable contribution.

Table 2: Ability variables contribution.

\begin{tabular}{c|c|c|c|c|c}
\hline Time & Money & Routine & Physical effort & Mental effort & Social Deviance \\
\hline+ & + & + & - & - & - \\
\hline
\end{tabular}

In this paper, the follow notation will be used:

$$
\begin{array}{lll}
\text { a1: Time } & \text { a2: Money } & \text { a3: Routine } \\
\text { a4: Physical effort } & \text { a5: Mental effort } & a_{6}: \text { Social deviance }
\end{array}
$$

The mathematical modeling for both axis shown a pattern on the axis formation process. However, the current FBM has six variables for each axis. Considering that the number of variables can change anytime in the future, it will be assumed that the quantity of positive and negative variables must be the same. This assumption is to avoid any bias on the axis formation process.

Thus, two distinct group can be formed. Let $\beta^{\alpha}$, with $\beta$ assuming M/A axis and $\alpha$ as $(+)$ for postive variables.

Negative variables follows analogous reasoning. Note that $x_{i}$ assumes values for all variables for both $m_{i}$ or $a_{i}$. Equation (2) shows the total sum for each presented group.

$$
\beta^{\alpha}=\sum_{i=1}^{n} x_{i}
$$

Let $n$, the amount of variables for both negative and positive values on the axis formation process follow the equation:

$$
\bar{\beta}=\beta^{+}-\beta^{-}=\sum_{i=1}^{\frac{n}{2}} x_{i}-\sum_{i=\frac{n}{2}+1}^{n} x_{i}
$$

Thus, the right hand side of Eq. (3) penalizes the resultant axis value since represents all negative contribution on the axis acquisition. However, since $\max \left(\beta^{+}\right)=3$ and $\max \left(\beta^{-}\right)=3$, for all variables defined in $I \in[0$, 1], the logistic function will be used in order to transform the linear combination of all variables into the FBM domain. Therefore, the final FBM axis formation equation is given by:

$$
\beta_{(\bar{\beta})}=\frac{1}{1+e^{-\bar{\beta}}}
$$

Marcus Guimaraes, Diana Adamatti and Leonardo Emmendorfer

An Agent-based Environment for Dynamic Positioning of the Fogg Behavior Model Threshold Line
ADCAIJ: Advances in Distributed Computing and Artifical Intelligence Journal Regular Issue, Vol. 7 N. 1 (2018), 67-76 eISSN: 2255-2863 - http://adcaij.usal.es Ediciones Universidad de Salamanca - CC BY NC DC 
Further analysis suggests that even if Eq. (3) results a null value, applying the logistic function it is possible to acquire a plausible FBM axis value since $\bar{\beta}=0 \Rightarrow \beta(\bar{\beta})=1 / 2$, which means a neutral value on the FBM domain.

\subsection{Threshold line}

The FBM Threshold Line is theoretical limit which informs all motivation and ability values capable of receive a simple stimulus called Trigger in order to achieve a target behavior, or promote a behavior change. In this paper, we believe that the threshold line can not be statically placed on the FBM plot. Its relation with all axis must be explicitly preserved and its allocation must be dynamic.

This action line has an intrinsic relation with further implementations of triggers. Hence, according to the dataset behavior, its correct positioning must be congruent with the data central tendency. Therefore, analyzing this action line function, as well its correct placement according to the dataset could result more efficient trigger approaches given its mathematical formalization, and not empirical as presented today.

Although not mentioned on the literature, by its shape, it is assumed that the threshold line mathematical function can be described by:

$$
f(x)=\frac{1}{x}
$$

on the $\mathrm{R}^{+}$domain.

The Kolmogorov mean will be used in order to demonstrate Eq. (5) properties. The Kolmogorov mean is a generalization of the most used means, such as arithmetic, geometric and harmonic (Slaev et al., 2013). By definition, if $f$ is a function that maps all real interval $I$, continuous and injective, the Kolmogorov mean is given by:

$$
M_{f}\left(x_{1}, \ldots, x_{n}\right)=f^{-1}\left(\frac{f\left(x_{1}\right)+\ldots+f\left(x_{n}\right)}{n}\right)
$$

Considering, if the domain is $\mathrm{R}^{+}$and the function to be used is $f(x)=1 / x$ then the Kolmogorov mean can be associated to the Harmonic mean (HM).

The use of the harmonic mean over other central tendencies tends to represent a more concise approach on the FBM context. It is known that the arithmetic mean is directly affected by extreme values. However, the harmonic mean is less sensible to such values.

The action line will be centered according to the dataset harmonic mean. This approach does not violates any FBM property. On the hand, ensures a coherent and robust way to relate the threshold line with triggers. This situation is neglected by the original Fogg theory. The important relation between the action line and triggers. That is the main reason why we believe it should be dynamic allocated according to the dataset central tendency. Therefore, the action line must be shifted. When properly placed, this threshold line has the power to show high levels of M/A observations. Consequently, positioned on the dataset harmonic mean promotes a reduction of observations above the line. This feature tends to avoid any bias since turns the action line extremely dependent on the dataset.

The function Eq.(5) has a center and it is given by $\mathrm{x}=\mathrm{y}=0.315$. Hence, it is possible to allocate this function according to the dataset harmonic mean as follows:

$$
\begin{aligned}
\text { Motivation } & =x+\text { HarmonicM ean }(M)-0.315 \\
\text { Ability } & =y+\text { HarmonicM ean }(A)-0.315
\end{aligned}
$$

\subsection{Agend-based simulation}

An agent-based simulation was chosen in order to simulated social interactions (Mangina and Carbo, 2010) in an environment capable of support all conditions for an FBM implementation. For this modeling process was considered three types of agents:

Marcus Guimaraes, Diana Adamatti and Leonardo Emmendorfer

An Agent-based Environment for Dynamic Positioning of the Fogg Behavior Model Threshold Line
ADCAIJ: Advances in Distributed Computing and Artifical Intelligence Journal Regular Issue, Vol. 7 N. 1 (2018), 67-76 elSSN: 2255-2863 - http://adcaij.usal.es Ediciones Universidad de Salamanca - CC BY NC DC 
- Regular agents are considered all ordinary people in a common social environment.

- Influencer type agent can be related to people with high level of motivation or ability. To preserve the robustness of the model, will be considered all observations above the dataset harmonic deviation (HD). Note that HD is analogous to the standard deviation, only considering the HM as central tendency on the evaluation process of data dispersion. This method was used to avoid any bias, since the action line use the harmonic mean.

- Extreme agents are those with distinct elevated level of motivation or ability. Adopting a statistical theory approach, it can be related to all observations above two harmonic deviations. These entities are capable of improve $\mathrm{M} / \mathrm{A}$ values on others with significant efficiency.

The interactive process between agents is defined according to the Algorithm 1.

An interaction counter is incremented on each agent contact. If an agent encounters a positive type, the variable pos is incremented. This method was adopted to measure the agent tendency. Once regular agents presents more positive tendency over negative, these type of agents will follow a positive influencer agent. Same reasoning can be extended to negative type agents.

However, when the agent contact one of its neighbors, a probability of being influenced is calculated. When this value results greater than the agent intrinsic value, then occurs the calculation for M/A. This method was adopted in order of similarity with real life situations. In practice, behavior change is not constant or some agent can be really difficult to persuade any change.

Given the agent type, motivation and ability will be calculated and incremented. Whenever an interaction occur between regular agents, the new M/A value will be random. The interaction between regular/influencer will improve values for $\mathrm{M} / \mathrm{A}$ by $10 \%$. This factor was implemented in order to highlight that influencer agents presents more persuasive power. Analogous reasoning for interactions with extreme agents. In that case, its persuasive power increases M/A by $25 \%$.

However, with this modeling process the system does not converge. Since all M/A values tends to change, in some point, given all agent interactions. This is an important aspect, since real life social interactions does not presents, necessarily, a converge behavior.

Figure 2 shows the implemented Netlogo environment for evaluation of all agent interactions. With this agent-based simulation process, both M/A axis values were acquired and the threshold line could be dynamically allocated according to the dataset center tendency corresponding to the harmonic mean.

Figure 2, grid, in black, shows all agent interactions. With all controls, located on the top, the user can calibrate the simulation, forcing more time, money or any other variable. The center plot shows all M/A values in real time and according to the simulation, the central tendency is calculated and the threshold line is allocated on the graphic. Some auxiliary graphics were also implemented in order to visualize the simulation dynamics.

\section{Results and Discussion}

Figure 3 shows that the original fixed FBM threshold line describes conceptually, trigger positions, but it is not efficient for practical purposes. No matter how the data changes, its fixed characteristic does not catch that data behavior and there is always a distance between the action line and the dataset central tendency. However, even

Marcus Guimaraes, Diana Adamatti and Leonardo Emmendorfer

An Agent-based Environment for Dynamic Positioning of the Fogg Behavior Model Threshold Line
ADCAIJ: Advances in Distributed Computing and Artifical Intelligence Journal Regular Issue, Vol. 7 N. 1 (2018), 67-76 elSSN: 2255-2863 - http://adcaij.usal.es Ediciones Universidad de Salamanca - CC BY NC DC 

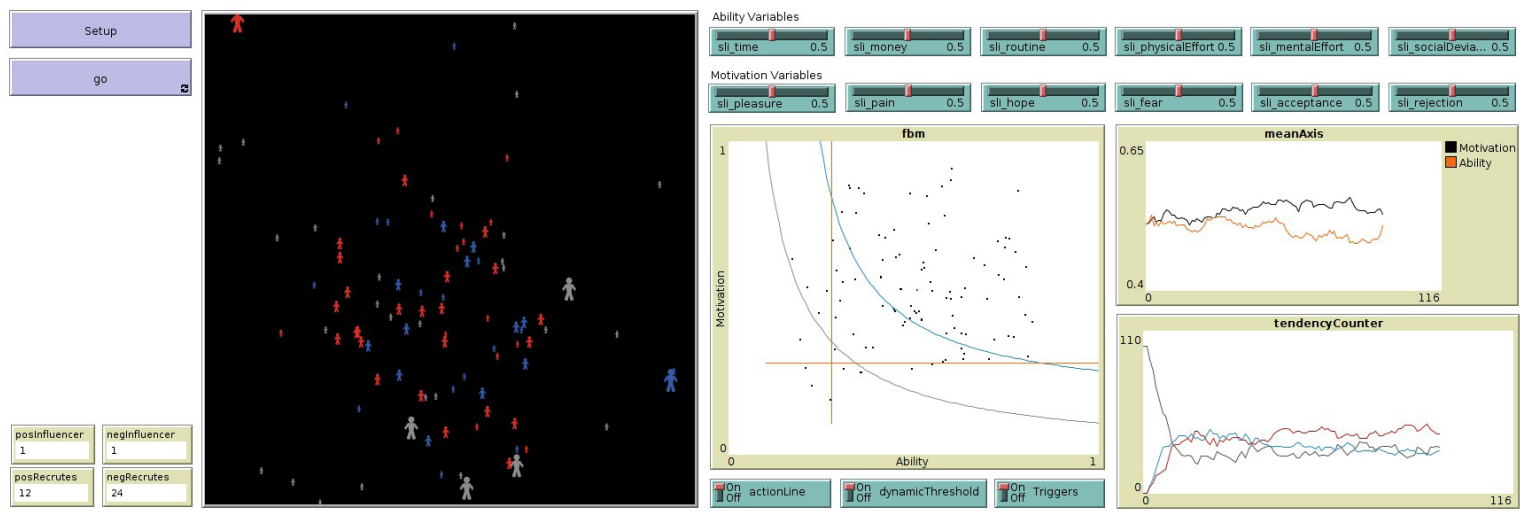

Figure 2: Agent-based simulation environment for FBM properties evaluation. Source: Author.

when the dynamic threshold line is used, related to the Kolmogorov mean, it represents a more reliable feature since its positioning is dynamic and captures all data change behavior.

The fixed threshold line underestimate the data when provides high amount of observation above the line. Therefore, the empirical threshold line, fixed, does not relate to the dataset. It is necessary to adopt another methodology and we propose the use of a dynamic threshold line based on the dataset central tendency as a more robust feature for evaluation of $\mathrm{M} / \mathrm{A}$ and future trigger implementations.
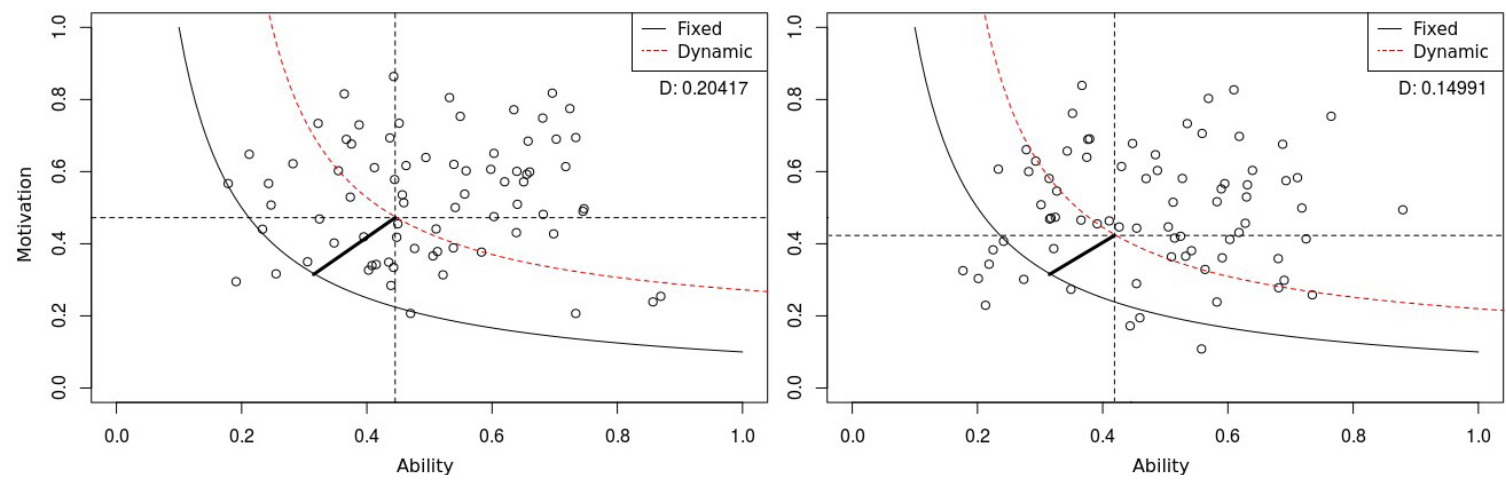

Figure 3: Comparison between fixed and dynamic threshold line and its power of detection and relation with data. Source: Author.

The dynamic threshold line is extremely dependent on the data since it is related to the dataset harmonic mean. We believe that this approach is really coherent since:

The distance (D in Fig. 3) will be null. The action line can act as a guide properly allocated on the data central tendency and provides more information about the data, when compared to the fixed threshold line feature.

The amount of observations above the action line is significantly reduced. This is a good approach because, in real applications, the detections of individuals above the threshold line implies a trigger to be implemented. Since the amount of unnecessary trigger is reduced, this means less financial costs in order of implementation such triggers.

Finally, using a dynamic threshold line demonstrates all its intrinsic relation to the data and triggers, since the type of triggers are related to the action line. We believe that dynamic relation ensures more efficient triggers, since data classification tends to be more accurate.

Therefore, the original, fixed, threshold line usage is not recommended, since it does not captures all data behavior, which it is a determinant factor on real life applications.

Marcus Guimaraes, Diana Adamatti and Leonardo Emmendorfer

An Agent-based Environment for Dynamic Positioning of the Fogg Behavior Model Threshold Line
ADCAIJ: Advances in Distributed Computing and Artifical Intelligence Journal Regular Issue, Vol. 7 N. 1 (2018), 67-76 elSSN: 2255-2863 - http://adcaij.usal.es Ediciones Universidad de Salamanca - CC BY NC DC 
With the agent-based simulation was able to evaluate $\mathrm{M} / \mathrm{A}$ value distribution. Table 3 show the sample mean distribution of M/A values according to three different methods: M/A Neutral, which means, without external influence on any variable. Using M Low/A random was considered a forced increment in one variable that makes the $\mathrm{M}$ final value. The main reason to use this approach was to verify the influence of the axis variables on the final axis value. On the decision making process, sometimes could be interesting to provide more time, or money, based on real applications, and see how this could affect the process outcome. Analogous reasoning when performed M High/A random.

Table 3: M/A sample distribution : ticks $=50.000(10 \times 5.000)$.

\begin{tabular}{c|c|c|c}
\hline & M/A Neutral & M Low/A random & M High/A random \\
\hline $\mathbf{M}$ & 0.52609 & 0.46141 & 0.58598 \\
\hline $\mathbf{A}$ & 0.51934 & 0.50162 & 0.49535 \\
\hline
\end{tabular}

When a disturbance factor is introduced on the system, in this case, changing the value of a variable $\left(m_{i}\right)$, it is possible to evaluate how the axis is affected. Was used the variable hope, for M Low and High, with values 0.1 and 0.9 respectively. This, initially, show that the axis modeling is consistent. The change of a variable alters the final axis behavior. In real FBM applications, the decision making responsible user could change any variable in order to evaluate the experiment.

Therefore, by given more hope, suppose it is a game system application, the user could evaluate this persuasive process in order to maintain the player playing the game even more. Analogous reasoning can be extended for variables such as money, time and so on, could improve the ability, and the persuasive process reach its goal easily.

With this in mind, our approach tends to be even more robust. When the system has disturbance, positive or negative, the dynamic threshold line shows the corresponding observations based on the data behavior itself. Hence, it can be used for more efficient trigger implementations when applied to persuasive technologies based processes.

\section{Conclusions}

In this paper, was presented a study on the axis formation process and the threshold line positioning based on the Fogg Behavior Model. According to the axis acquisition generalization, the mathematical modeling shows a similarity for both axis considering all variables. No previous study was found on the literature considering the axis acquisition using all variables.

It was possible to evaluate the threshold line behavior. Our analysis suggests that, in order to provide a robust feature for data classification and trigger implementation, the action line must not be fixed, as originally presented, but dynamically allocated based on the dataset central tendency. We used the Kolmogorov mean as a mathematical support for link the threshold line with the data harmonic mean.

Results showed that using the dynamic threshold line, all FBM properties are fulfilled and, given its intrinsic relation, better trigger implementations can be further performed. Therefore, as soon the data is updated, the dynamic threshold line detects this new pattern and provides a new location, where observations can be labeled and receive the correct stimuli. This paper tends to contribute with a theoretical approach providing better reasoning for future persuasive technologies implementations based on the Fogg Behavior Model.

In future works, we would like to evaluate different triggers when identified by the dynamic threshold line in real time acquisition data agent-based system.

Marcus Guimaraes, Diana Adamatti and Leonardo Emmendorfer

An Agent-based Environment for Dynamic Positioning of the Fogg Behavior Model Threshold Line
ADCAIJ: Advances in Distributed Computing and Artifical Intelligence Journal Regular Issue, Vol. 7 N. 1 (2018), 67-76 elSSN: 2255-2863 - http://adcaij.usal.es Ediciones Universidad de Salamanca - CC BY NC DC 


\section{References}

Azar, K., Lesser, L., and Stephens, J., 2013. Mobile applications for weight management theory-based content analysis. American Journal of Preventive Medicine, 45(5):583-589.

Bergmans, A. and Shahid, S., 2013. Reducing speeding behavior in young drivers using a persuasive mobile application. Human-Computer Interaction: Applications and Services: 15th International Conference, HCI International Las Vegas, NV, USA, Springer Berlin Heidelberg, pages 541-550.

Fogg, B. J., 2003. Persuasive Technology: Using Computers to Change What We Think and Do. Morgan Kaufmann, San Francisco.

Fogg, B. J., 2009. A behavior model for persuasive design. Persuasive '09 Proceedings of the 4th International Conference on Persuasive Technology, ACM, New York, (40).

Hillard, R., 2010. Information-Driven Business: How to Manage Data and Information for Maximum Advantage.

John Wiley \& Sons.

Hogan, K., 2004. The Psychology of Persuasion. How to persuade others to your way of thinking. Pelican Publishing Company, Gretna.

Hongqi, W., Zongwei, L., ZhangYuyu, T., and Wu, Y., 2013. A pervasive technology approach to social trustworthiness. Communications in Computer and Information Science, 320:242-249.

Larson, J., 2014. The invisible, manipulative power of persuasive technology. http://www.psmag.com/ nature- and-technology/captology-fogg-invisible-manipulative-power-persuasive-technology-81301.

Mangina, E. and Carbo, J., 2010. Agent-Based Ubiquitous Computing. Atlantis Ambient and Pervasive Intelligence. Atlantis Press.

Rahman, H., 2016. Human Development and Interaction in the Age of Ubiquitous Technology. Advances in Human and Social Aspects of Technology, IGI Global.

Shannon, R., 1975. Simulation: A survey with research suggestions. AIIE Transactions, Taylor and Francis Online, 7(3):289-301.

Slaev, V., Chunovkina, A., and Mironovsky, L., 2013. Metrology and Theory of Measurement. De Gruyter Studies in Mathematical Physics. De Gruyter.

Tsai, M., Chang, Y., and Kao, C., 2015. The effectiviness of a flood protection computer game for disaster education. Visualization in Engineering.

Uhrmacher, A. M. and Weyns, D., 2009. Multi-Agent Systems: Simulation and Applications. Computational Analysis, Synthesis, and Design of Dynamic Systems, CRC Press.

Wessels, W., 2010. Practical Reliability Engineering and Analysis for System Design and Life-Cycle Sustainment. CRC Press.

Marcus Guimaraes, Diana Adamatti and Leonardo Emmendorfer

An Agent-based Environment for Dynamic Positioning

of the Fogg Behavior Model Threshold Line
ADCAIJ: Advances in Distributed Computing and Artifical Intelligence Journal Regular Issue, Vol. 7 N. 1 (2018), 67-76 eISSN: 2255-2863 - http://adcaij.usal.es Ediciones Universidad de Salamanca - CC BY NC DC 


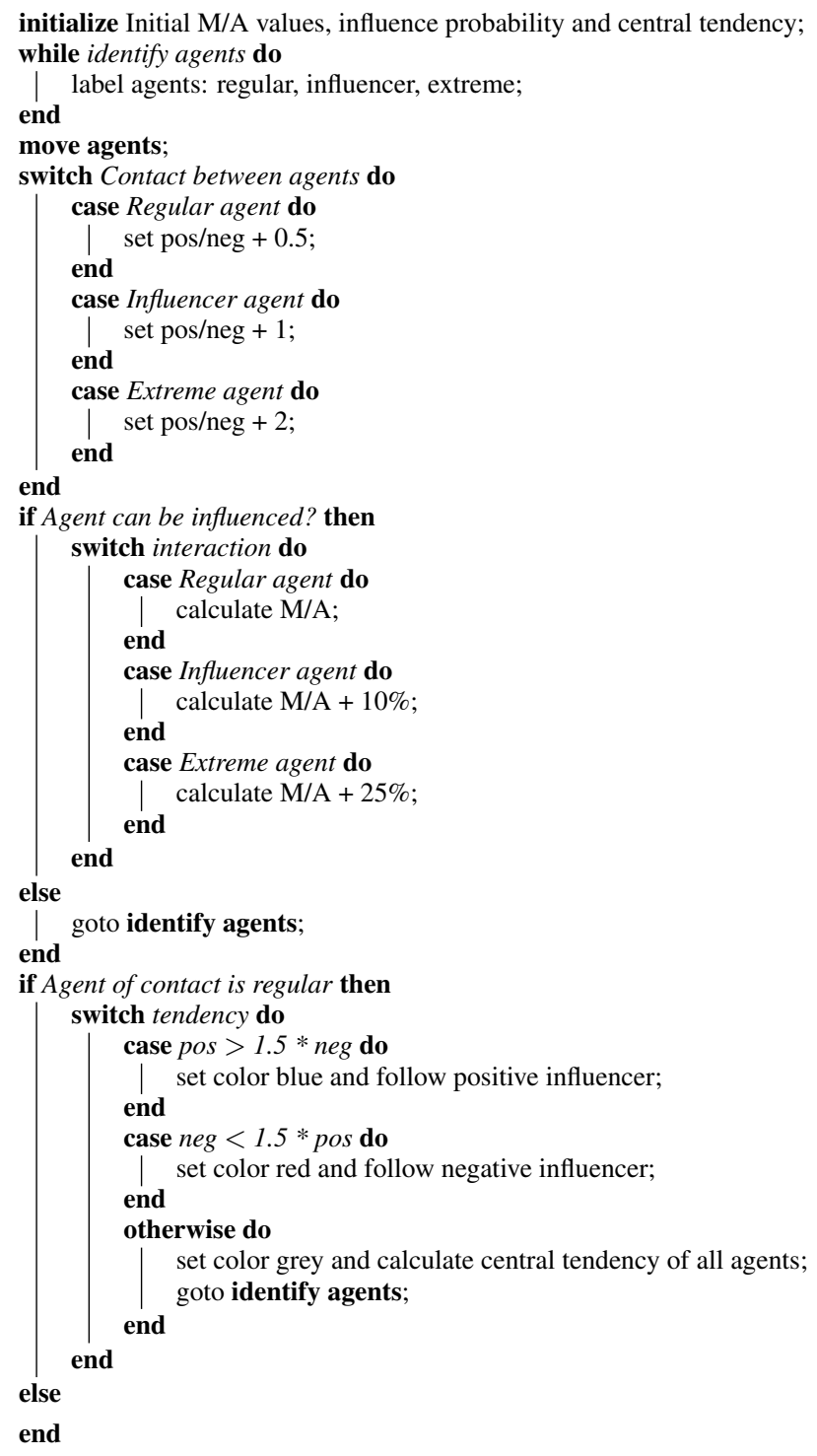

Algorithm 1: Agent-based interaction for M/A evaluation and Threshold line positioning. Source: Author.

Marcus Guimaraes, Diana Adamatti and Leonardo Emmendorfer

An Agent-based Environment for Dynamic Positioning of the Fogg Behavior Model Threshold Line
ADCAIJ: Advances in Distributed Computing and Artifical Intelligence Journal Regular Issue, Vol. 7 N. 1 (2018), 67-76 eISSN: 2255-2863 - http://adcaij.usal.es Ediciones Universidad de Salamanca - CC BY NC DC 